\title{
Linear Regression Technique for Improvement of Feet Position Estimation during Standing Balance Using a Cane with Millimeter Wave Radar
}

\author{
Ibai Gorordo Fernandez ${ }^{1 *}$, Kodai Kitagawa ${ }^{1}$, Kawthar Abdul Rahman ${ }^{2}$, Azura \\ Che Soh ${ }^{3}$, Alpha Agape Gopalai ${ }^{4}$, Siti Anom Ahmad ${ }^{2}$ and Chikamune Wada ${ }^{1}$ \\ ${ }^{1}$ Deparment of Life Science and Systems Engineering, Kyushu Institute of Technology, 2-4 Hibikino, \\ Wakamatsu-ku, Kitakyushu-shi, Fukuoka 808-0196, Japan \\ ${ }^{2}$ Malaysian Research Institute on Ageing (MyAgeing), Universiti Putra Malaysia 43400 Serdang, \\ Selangor, Malaysia \\ ${ }^{3}$ Department of Electrical and Electronic Engineering, Faculty of Engineering, Universiti Putra Malaysia \\ 43400 Serdang, Selangor, Malaysia \\ ${ }^{4}$ School of Engineering, Monash University of Malaysia, Bandar Sunway 46150 Selangor, Malaysia
}

\begin{abstract}
It is estimated that one in three seniors fall at least once a year. Falls are a global problem for the elderly that affects their quality of life and poses a great risk. In our research, we are trying to develop a system that could prevent falls by estimating the fall risk in real time. The system would measure the balance of the user by measuring the position of the Center of Gravity inside the Base of Support. In our

ARTICLE INFO

\section{Article history:}

Received: 10 February 2020

Accepted: 13 November 2020

Published: 31 December 2020

DOI: https://doi.org/10.47836/pjst.28.S2.06

\section{E-mail addresses:}

ibai.gorordo@hotmail.com (Ibai Gorordo Fernandez) kitagawakitagawa156@gmail.com (Kodai Kitagawa)

kawthar.ar@gmail.com (Kawthar Abdul Rahman) azuracs@upm.edu.my (Azura Che Soh)

alpha.agape@monash.edu (Alpha Agape Gopalai)

sanom@upm.edu.my (Siti Anom Ahmad)

wada@brain.kyutech.ac.jp (Chikamune Wada)

*Corresponding author previous research, we presented a system with a millimeter wave radar attached to a cane to measure the area of the Base of Support. However, the obtained results for the foot position estimation error were significantly worse than similar studies. One of the reasons was that the sensor was not really estimating the position of the feet but the position of the lower legs. Therefore, in this research we present a correction model to improve the feet position estimation. The proposed model was able to reduce the foot position estimation RMSE from 54
\end{abstract}


$\mathrm{mm}$ down to $34 \mathrm{~mm}$, which is closer to the results of other similar studies measuring the position of the feet.

Keywords: Balance analysis, falls, feet position estimation, millimeter wave radar

\section{INTRODUCTION}

Falls are a major health risk for the elderly. It is estimated that about one in three seniors falls at least once a year (Rubenstein \& Josephson, 2002). Moreover, according to the same study, more than half of these seniors suffered multiple falls each year. Even though elderly fall rates vary depending on the country, e.g. 20\% in Japan (Sakita et al., 2015) or $32.8 \%$ in Malaysia (Kioh \& Rashid, 2018), falls pose a great risk for the elderly. It is estimated that falls are the cause of over 400,000 deaths worldwide every year and over $50 \%$ of those fallers were aged 60 years or older (Jagnoor et al., 2013).

Furthermore, falls also affect severely to the quality of life of the elderly. Falls in the elderly often result in injuries that lead to mobility impairments. For this reason, assistive devices such as cane or walker are commonly prescribed to the elderly population. It is estimated that there are over 4 million cane users only in the United States (Bateni \& Maki, 2005). The use of these assistive devices helps mobility impairment patients to recover some mobility by improving the stability of the user. This is achieved by increasing the area of the Base of Support (BoS), which refers to the area between the contact points of the body with the ground. As long as the Center of Gravity $(\mathrm{CoG})$ is kept inside the BoS, stable balance is maintained. That is why by increasing the area of the BoS, for example using assistive devices, it increases the stable range of movement of the $\mathrm{CoG}$ inside the BoS. However, as recent studies show, assistive device users have a higher injury fall risk compared to non-users (West et al., 2015).

Due to the terrible consequences of falls, many studies have tried to develop a system to detect falls. The use of these devices can reduce the adverse consequences of falls by providing rapid treatment as soon as a fall is detected (Igual et al., 2013). Most of the developed fall detection systems used one inertial sensor (Pierleoni et al., 2015) or multiple inertial sensors (Nyan et al., 2008) attached to the body to detect falls. Similarly, Lan et al. (2010) attached a wireless inertial sensor to a single tip cane to detect fall events using a multi-stage thresholding algorithm.

Recently, a higher number of studies have developed ambient sensor-based fall detection systems. These systems use sensors fixed in different parts of a room to detect falls inside the field of view of the sensors. Different types of sensors have been used in these kinds of systems. For example, Amin et al. (2016) used a doppler radar to detect different types of falls, while Yang et al. (2016) used a depth camera to detect any fall 
that happened inside the camera's field of view. Similarly, De Miguel et al. (2017) also developed a fall detection system using a low cost camera.

On the other hand, multiple studies have developed balance assessment systems to predict the risk of falls over the long term. These types of systems mostly use force platforms to estimate the movement of the $\mathrm{CoG}$ or the Center of Pressure $(\mathrm{CoP})$ during standing balance to assess the balance of the patients (Clark et al., 2010). Another common method is the use of an inertial sensor attached to the body to estimate the postural sway during stance as an indicator of stability (Mancini \& Horak, 2010). The limitation of these systems is that even though they can accurately estimate the fall risk in the future, due to the unpredictability of falls, these systems cannot be used to measure the fall risk in real time.

However, a few studies have tried to develop real time fall risk assessment systems. The objective of our research is to develop a real time fall risk assessment system by measuring the position of the $\mathrm{CoG}$ inside the $\mathrm{BoS}$ in daily life. Measuring the fall risk in real time could help to prevent falls by alerting the user when the risk of fall is high. Van Meulen et al. (2016) developed a similar system for balance analysis by placing multiple sensors underneath a pair of sandals. However, due to the high number of sensors, each shoe had two 3D force/moment sensors, two inertial sensors and an ultrasonic sensor; the shoes were heavy $(1 \mathrm{~kg})$ and could be dangerous for the elderly due the extra sole height. For this reason, in our previous study (Fernandez \& Wada, 2019), we developed a system to estimate the area of the BoS by using a millimeter wave radar attached to a cane. By attaching the sensor to normal cane, the system could be used without the need to wear any special shoes or clothes. However, the obtained results were worse than other similar studies.

For this reason, in this research we present a correction model to improve the feet position estimation. The proposed correction model takes the estimated lower leg position obtained from our previous system and outputs a corrected feet position.

\section{MATERIALS AND METHODS}

\section{Previous Study}

The data used in this research was obtained in our previous study (Fernandez \& Wada, 2019). In that study, 3 young male subjects (age $24.67 \pm 2.08$ ) with no previous balance or gait impairments participated in the experiment. The subjects were asked to stand for 3 seconds while placing the right foot in 9 different locations based on the results from previous studies (Kuan et al., 1999). The 9 different foot locations corresponded with three different stance lengths and three different stance width combinations as shown in Table 1.

In our previous study, we attached a millimeter wave radar (AWR1642, Texas Instruments) to a single tip cane at a height of $10 \mathrm{~cm}$ and pointing towards the feet. The radar was used to measure the $2 \mathrm{D}$ horizontal position of the reflected points inside its 
$110 \mathrm{deg}$. field of view (FoV) at $20 \mathrm{~Hz}$. Then, our developed algorithm was used on the reflected point cloud to extract the $2 \mathrm{D}$ position of the centroids for the left and right feet as shown in Figure 1.

Table 1

Stance length and width values for the 9 different foot locations in the experiment

\begin{tabular}{lll}
\hline Pattern Number & Stance Length $(\mathrm{cm})$ & Stance Width $(\mathrm{cm})$ \\
\hline 1 & 18.8 & 23.5 \\
2 & 18.8 & 19.6 \\
3 & 18.8 & 15.7 \\
4 & 28.6 & 23.5 \\
5 & 28.6 & 19.6 \\
6 & 28.6 & 15.7 \\
7 & 38.4 & 23.5 \\
8 & 38.4 & 19.6 \\
9 & 38.4 & 15.7 \\
\hline
\end{tabular}

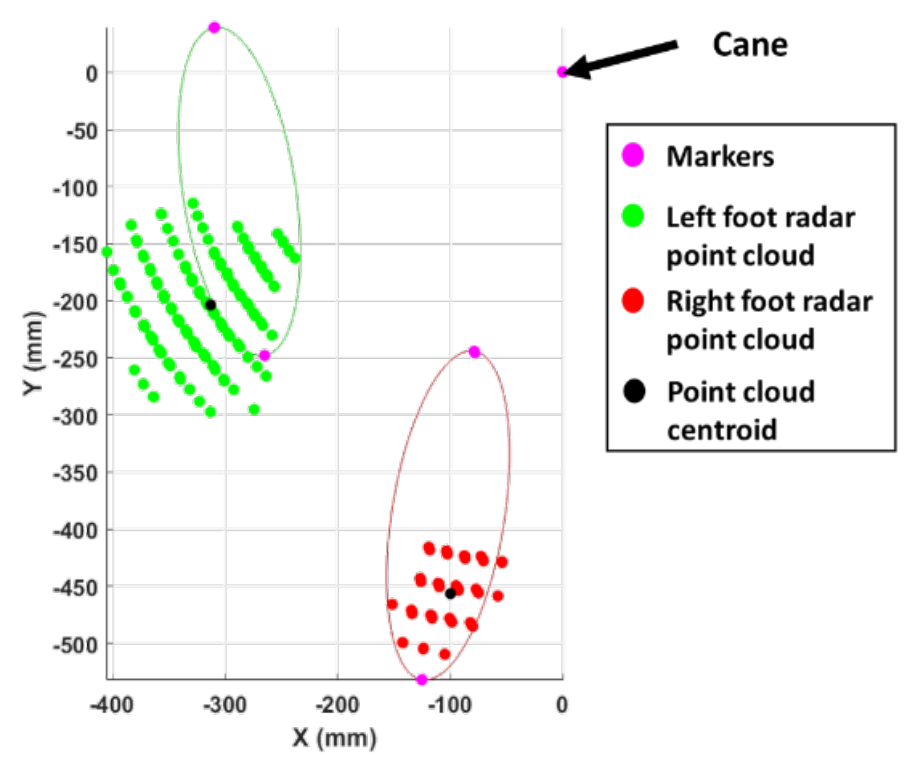

Figure 1. Example of the point cloud data and centroids obtained from the radar as well as the position of the reflected markers 
On the other hand, in order to validate the results from the radar, we used an optical motion capture system with 8 infrared cameras (FLEX 13, Oprtitrack). The motion capture system tracked the $3 \mathrm{D}$ position of 5 reflective markers placed in the shoes and the cane. As shown in Figure 1, two markers were placed in each shoe, one at the front and one at the rear, and another marker was placed on the tip of the cane. The motion capture system captured the position of the markers at $100 \mathrm{~Hz}$, but then the captured 3D position was downsampled to $20 \mathrm{~Hz}$ to match with the sampling frequency of the radar. As a result, a total of 3851 samples were obtained both from the motion capture system as well as from the millimeter wave radar.

As a result, our previous system was able to calculate the area of the Base of Support with a RMSE value of $92.04 \mathrm{~cm}^{2}$, corresponding with the $7 \%$ of the total Base of the Support area. Also, the foot distance error was of $5.4 \mathrm{~cm}$, it was significantly worse than the error of $2.26 \mathrm{~cm}$ obtained in a similar study that used a shoe type measurement device (Widodo \& Wada, 2017).

One of the reasons for these worse results was that the reflected points were not representing the position of the feet, but the position of the lower leg as shown in Figure 2 with the reflected points in blue. This happened because the lower leg had a larger surface area than the shoes, therefore more points were reflected on the lower leg compared to the points reflected on the shoes. However, since the objective of our research was to estimate the fall risk using the position of the feet, we needed to develop a model to obtain the correct position of the feet.

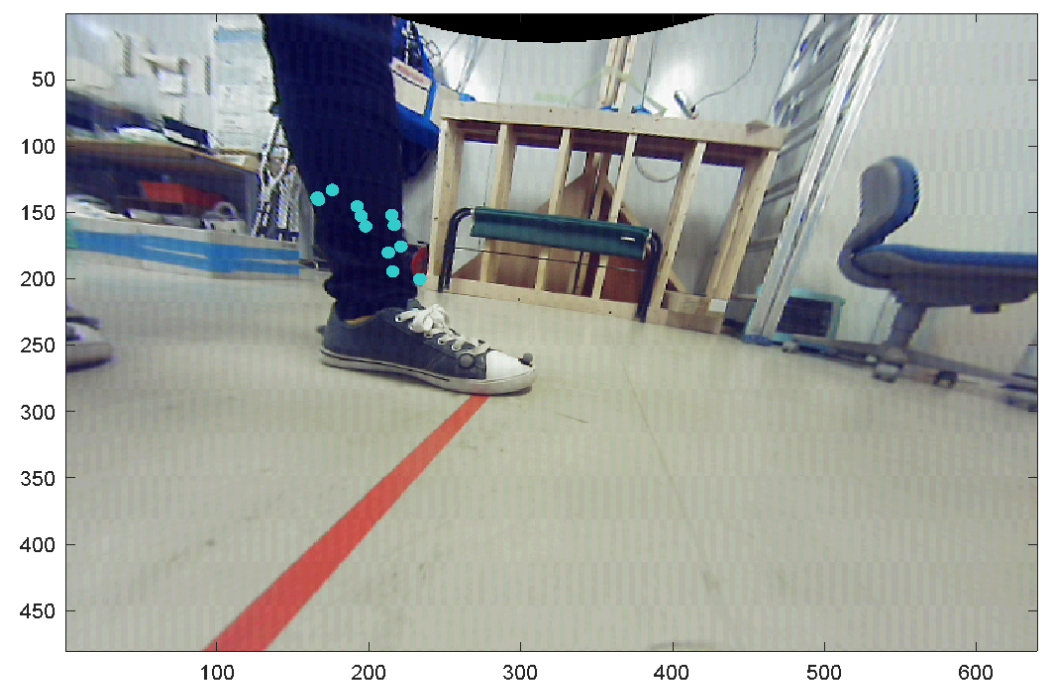

Figure 2. Example of the position of the points obtained from the radar (blue) that reflected on the lower leg 


\section{Correction Model}

For that purpose, in this research we developed a correction model that took the centroids (red points in Figure 3), that represented the 2D position of the lower legs, and estimated the correction distances. The correction (horDist ${ }_{\mathrm{L}}$, verDist $\mathrm{L}_{\mathrm{L}}$, horDist $_{\mathrm{R}}$ and verDist $_{\mathrm{R}}$ ) represented the horizontal and vertical distances from the centroid to the heel marker as shown in Figure 3.

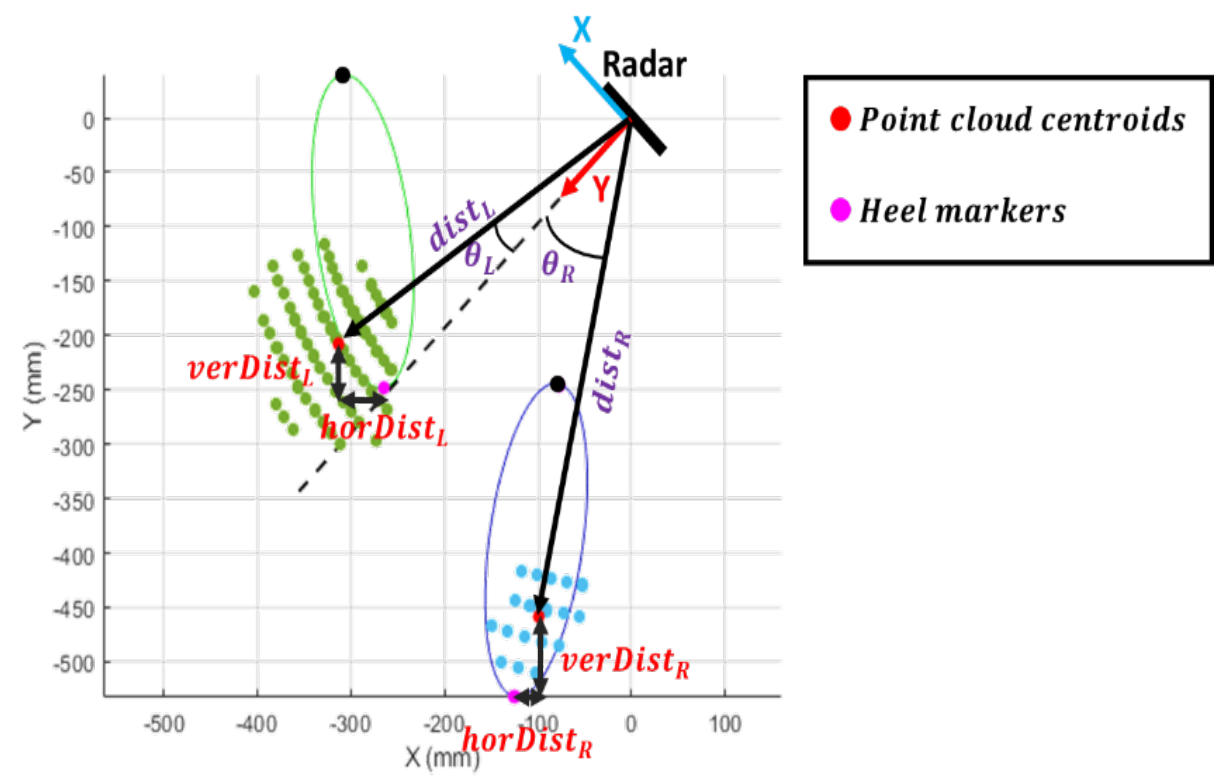

Figure 3. Diagram describing the input $\left(\theta_{\mathrm{L}}, \theta_{\mathrm{R}}\right.$, dist $\mathrm{L}_{\mathrm{L}}$, and dist $\left.t_{\mathrm{R}}\right)$ and output (horDist $\mathrm{L}_{\mathrm{L}}$, verDist $_{\mathrm{L}}$, horDist $_{\mathrm{R}}$ and verDist $t_{R}$ ) of the correction model

On the other hand, the position of the centroids was represented in polar coordinates where $\theta_{\mathrm{L}}$ and $\theta_{\mathrm{R}}$ were the angles from the $\mathrm{Y}$ axis of the radar for the left and right centroids respectively; and similarly, dist $_{L}$ and dist ${ }_{R}$ were the distances from the radar to the centroids as shown in Figure 3. Also, for simplicity, instead of creating one model for the 4 correction distances, we generated 4 different models, one for each correction distance.

\section{Parameter Selection}

In order to create each of the 4 correction models, it was necessary to decide which parameter was going to be selected as input for each model. For that purpose, instead of only trying to use the position of the centroids $\left(\theta_{\mathrm{L}}, \theta_{\mathrm{R}}\right.$, dist $_{\mathrm{L}}$, and dist $\left.\mathrm{R}_{\mathrm{R}}\right)$, another 56 different parameters were extracted from those 4 parameters. Then, in order to select the best 
parameter for each model, the Pearson correlation between each of the extracted parameters and each of the correction distances was calculated.

Once all the correlation values were extracted, the parameter with the highest absolute Pearson correlation was selected for each correction model. The Pearson correlation represents the linear correlation between the two variables. Therefore, the parameter with the highest Pearson correlation could provide more information about the desired correction distance than the rest of the parameters.

\section{Linear Regression Model}

Finally, in order to obtain each of the correction models, a linear regression model was used to estimate each correction distance based on the values from its corresponding selected parameter. Figure 4 depicts the linear fits for each of the 4 correction distances as well as the $95 \%$ confidence interval regions.

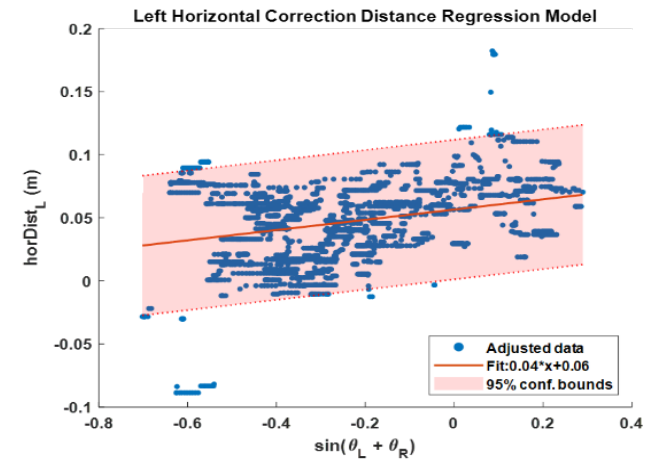

(a)

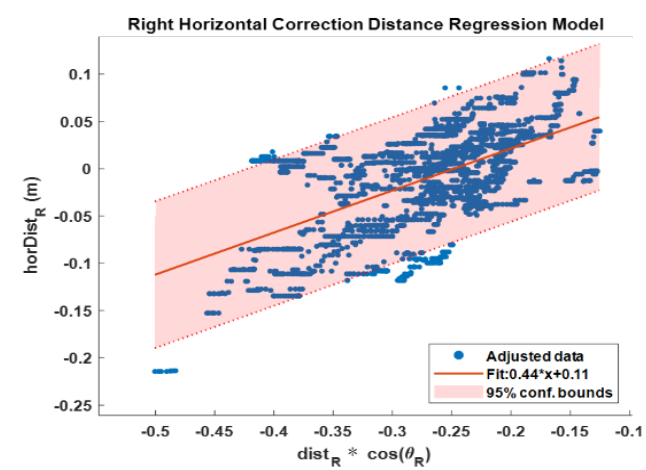

(c)

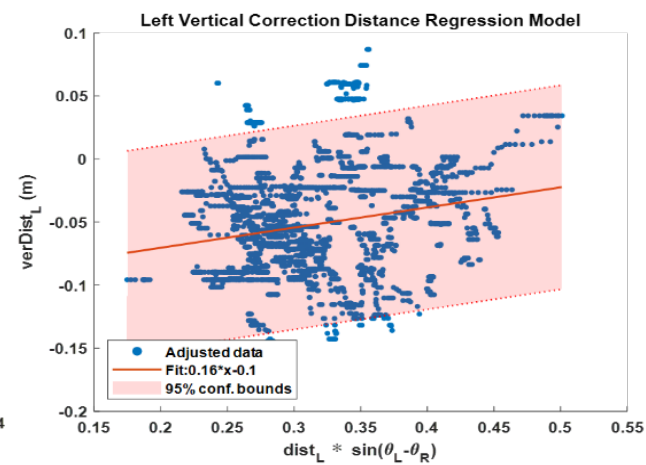

(b)

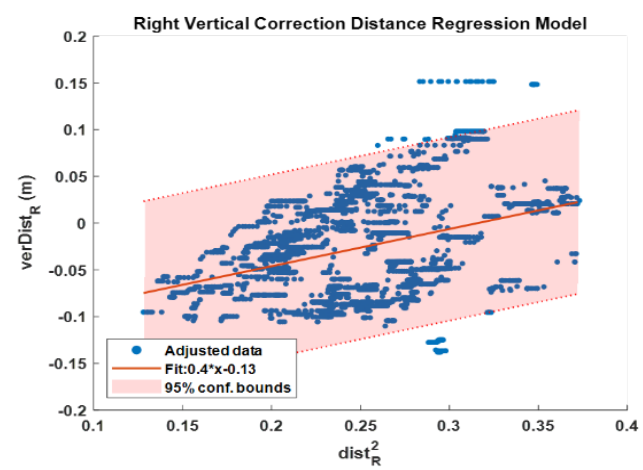

(d)

Figure 4. Linear regression models for each of the 4 correction distances: (a) horDist ${ }_{\mathrm{L}}$, (b) verDist $\mathrm{L}_{\mathrm{L}}$, (c) horDist $_{R}$, (d) verDist . $_{\text {. }}$ 


\section{RESULTS AND DISCUSSIONS}

Table 2 summarizes the results for the correction models. The table shows the Pearson correlation between each correction distance and its selected parameter, the linear fit function, as well as the RMSE value for the linear fit. As it can be observed, the correction model for the verDist $t_{R}$ had the highest RMSE fit. A higher RMSE fit indicates that when using the correction model to correct the feet position, a higher error can be expected. One of the reasons why the results for the right foot were worse than those for the left foot, was that while the left foot was in front of the radar, the right foot was near the limit of the field of view of the radar. Because of this, in some cases, the number of reflected points on the right lower leg were scarce and the noise was higher than for the left side.

Next, once the 4 linear correction models were generated, these models were used to estimate the correction distances for each of the measurements. Then, the correction distances were used to update the previous estimated centroid positions in order to estimate the position of the heels. As a result, we were able to reduce the foot position estimation from $62.66 \mathrm{~cm}$ to $35.31 \mathrm{~mm}$ for the left foot (43.65\% error reduction) and from 54.76 to $45.6 \mathrm{~mm}$ for the right foot (16.73\% error reduction). The reason why the error reduction for the left foot position estimation was worse than for the right foot both in absolute and in relative terms was because regression fit error was also worse for the correction models of the right foot than of the left foot as shown in Table 2.

Table 2

Summary of the results for the correction models generation

\begin{tabular}{lllll}
\hline $\begin{array}{l}\text { Correction } \\
\text { Distance }\end{array}$ & $\begin{array}{l}\text { Selected } \\
\text { Parameter }\end{array}$ & Pearson Corr. & $\begin{array}{l}\text { Fit } \\
\text { Function }\end{array}$ & $\begin{array}{l}\text { Fit RMSE } \\
(\mathrm{mm})\end{array}$ \\
\hline horDist $_{\mathrm{L}}$ & $\sin \left(\theta_{\mathrm{L}}+\theta_{\mathrm{R}}\right)$ & 0.31 & $\mathrm{y}=0.04 *^{*}+0.06$ & 28.2 \\
verDist $_{\mathrm{L}}$ & $\operatorname{dist}_{\mathrm{L}} * \sin \left(\theta_{\mathrm{L}}-\theta_{\mathrm{R}}\right)$ & 0.2 & $\mathrm{y}=0.16^{*} \mathrm{x}-0.1$ & 41.1 \\
horDist $_{\mathrm{R}}$ & $\operatorname{dist}_{\mathrm{L}} * \cos \left(\theta_{\mathrm{R}}\right)$ & 0.6 & $\mathrm{y}=0.44 *_{\mathrm{x}}+0.11$ & 39.5 \\
verDist $_{\mathrm{R}}$ & $\left(\text { dist }_{\mathrm{R}}\right)^{2}$ & 0.35 & $\mathrm{y}=0.4 *_{\mathrm{x}}-0.13$ & 49.9 \\
\hline
\end{tabular}

Similarly, Table 3 summarizes the stance length and stance width RMSE values before and after applying the correction model. As it can be observed, even though the error reduction for the stance length estimation was smaller than $1 \mathrm{~cm}$, the RMSE value for the stance width was reduced to almost half after applying the correction model.

Overall, the RMSE value for the distance between the feet (Foot Distance RMSE in Table 3) was reduced from $54.39 \mathrm{~mm}$ down to $34.19 \mathrm{~mm}$ after applying the correction models, representing a $37.14 \%$ error reduction. The obtained foot distance RMSE was 
closer to the error of $22.6 \mathrm{~mm}$ obtained by Widodo and Wada (2017). Even though the current results are still worse than those of previous studies, our current system can estimate the feet position without the need to wear special heavy shoes, as the weight of our sensor system is only $38 \mathrm{~g}$.

Table 3

Summary of the stance length and stance width RMSE values before and after applying the correction models

\begin{tabular}{llll}
\hline & $\begin{array}{l}\text { Stance Width RMSE } \\
(\mathrm{mm})\end{array}$ & $\begin{array}{l}\text { Stance Length } \\
\text { RMSE }(\mathrm{mm})\end{array}$ & $\begin{array}{l}\text { Foot Distance } \\
\text { RMSE }(\mathrm{mm})\end{array}$ \\
\hline Before Correction & 63.46 & 43.47 & 54.39 \\
Corrected & 33.44 & 34.93 & 34.19 \\
\hline
\end{tabular}

\section{CONCLUSIONS}

In this paper, a correction model for foot position estimation is presented. The proposed model takes the raw centroids of the radar point cloud data and using a linear regression model estimates the correct heel position. The proposed model was evaluated using the data from our previous study and the obtained results showed that the model was able to reduce the feet position estimation almost down to half.

However, the proposed model was only tested on data during standing balance. Also, one of the limitations of this study is the reduced number of participants. In the future, we will test our sensor with a higher number of participants as well as using data taken also during gait experiments in order to observe how the system works also during dynamic movements. For that purpose, it is necessary to know the orientation of the radar in order to transform the radar point cloud data from the local axes of the radar to the world coordinates. That is why we will also add an inertial sensor to our system in order to estimate the orientation and movement of the cane during gait.

\section{ACKNOWLEDGEMENT}

This research was partly funded by the Collaboration Program between Kyushu Institute of Technology and Universiti Putra Malaysia.

\section{REFERENCES}

Amin, M. G., Zhang, Y. D., Ahmad, F., \& Ho, K. C. D. (2016). Radar signal processing for elderly fall detection: The future for in-home monitoring. IEEE Signal Processing Magazine, 33(2), 71-80. doi:10.1109/ msp.2015.2502784 
Ibai Gorordo Fernandez, Kodai Kitagawa, Kawthar Abdul Rahman, Azura Che Soh, Alpha Agape Gopalai, Siti Anom Ahmad and Chikamune Wada

Bateni, H., \& Maki, B. E. (2005). Assistive devices for balance and mobility: Benefits, demands, and adverse consequences. Archives of Physical Medicine and Rehabilitation, 86(1), 134-145. doi:10.1016/j. apmr.2004.04.023

Clark, R. A., Bryant, A. L., Pua, Y., McCrory, P., Bennell, K., \& Hunt, M. (2010). Validity and reliability of the Nintendo Wii Balance Board for assessment of standing balance. Gait and Posture, 31(3), 307-310. doi:10.1016/j.gaitpost.2009.11.012

De Miguel, K., Brunete, A., Hernando, M., \& Gambao, E. (2017). Home camera-based fall detection system for the elderly. Sensor, 17(12), 1-21. doi:10.3390/s17122864

Fernandez, I. G., \& Wada, C. (2019, March 12-14). Cane with millimeter wave radar for base of support measurement. In 2019 IEEE 1st Global Conference on Life Sciences and Technologies, LifeTech (pp. 133-136). Osaka, Japan.

Igual, R., Medrano, C., \& Plaza, I. (2013). Challenges, issues and trends in fall detection systems. BioMedical Engineering Online, 12(1), 2-24. doi:10.1186/1475-925x-12-66

Jagnoor, J., Keay, L., \& Ivers, R. (2013). A slip and a trip? Falls in older people in Asia. Injury, 44(6), 701-702. doi: 10.1016/j.injury.2013.03.009

Kioh, S. H., \& Rashid, A. (2018). The prevalence and the risk of falls among institutionalised elderly in Penang, Malaysia. Medical Journal of Malaysia, 73(4), 212-219.

Kuan, T. S., Tsou, J. Y., \& Su, F. C. (1999). Hemiplegic gait of stroke patients: The effect of using a cane. Archives of Physical Medicine and Rehabilitation, 80(7), 777-784. doi:10.1016/s0003-9993(99)90227-7

Lan, M., Nahapetian, A., Vahdatpour, A., Au, L., Kaiser, W., \& Sarrafzadeh, M. (2010, September 10-12). SmartFall: An automatic fall detection system based on subsequence matching for the smartcane. In Conference on Body Area Networks (pp. 1-8). Los Angeles, California.

Mancini, M., \& Horak, F. B. (2010). The relevance of clinical balance assessment tools to differentiate balance deficits. European Journal of Physical and Rehabilitation Medicine, 46(2), 239-248.

Nyan, M. N., Tay, F. E. H., \& Murugasu, E. (2008). A wearable system for pre-impact fall detection. Journal of Biomechanics, 41(16), 3475-3481. doi:10.1016/j.jbiomech.2008.08.009

Pierleoni, P., Belli, A., Palma, L., Pellegrini, M., Pernini, L., \& Valenti, S. (2015). A high reliability wearable device for elderly fall detection. IEEE Sensors Journal, 15(8), 4544-4553. doi:10.1109/JSEN.2015.2423562

Rubenstein, L. Z., \& Josephson, K. R. (2002). The epidemiology of falls and syncope. Clinics in Geriatric Medicine, 18(2), 141-158. doi:10.1016/s0749-0690(02)00002-2

Sakita, M., Murakami, S., Saito, T., \& Kumagai, S. (2015). Falls and fall prevention in elderly people: Summary of recent reviews. Japanese Journal of Health Promotion and Physical Therapy, 4(4), 161169. doi:10.9759/hppt.4.161

Van Meulen, F. B., Weenk, D., Buurke, J. H., Van Beijnum, B. J. F., \& Veltink, P. H. (2016). Ambulatory assessment of walking balance after stroke using instrumented shoes. Journal of Neuroengineering and Rehabilitation, 13(1), 1-10. doi:10.1186/s12984-016-0146-5 
West, B. A., Bhat, G., Stevens, J., \& Bergen, G. (2015). Assistive device use and mobility-related factors among adults aged $\geq 65$ years. Journal of Safety Research, 55, 147-150. doi:10.1016/j.jsr.2015.08.010

Widodo, R. B., \& Wada, C. (2017). Artificial neural network based step-length prediction using ultrasonic sensors from simulation to implementation in shoe-type measurement device. Journal of Advanced Computational Intelligence and Intelligent Informatics, 21(2), 321-329. doi:10.20965/jaciii.2017.p0321

Yang, L., Ren, Y., \& Zhang, W. (2016). 3D depth image analysis for indoor fall detection of elderly people. Digital Communications and Networks, 2(1), 24-34. doi:10.1016/j.dcan.2015.12.001 
